\title{
Mollusques pulmonés d'eau douce basommatophores, vecteurs au Tchad d'affections parasitaires du bétail ; leur élevage au laboratoire
}

\author{
par E. BIRGI (*) et M. GRABER (**)
}

\begin{abstract}
RÉSUMË
Les auteurs après avoir souligné l'importance pour le bétail adulte des zones sahélo-soudaniennes du Tchad des affections parasitaires dues à certains trématodes hépatiques, stomacaux et veineux (Fosciola, Paramphistomum, Carmyerius et Schistosomes), décrivent une méthode simple ef pratique permettant, dans des conditions climatiques très dures, l'élevage des principaux mollusques vecteurs.

lis donnent également quelques indications concernant le cycle saisonnier de $B$. forskolii, B. jousseaumei ; B. truncotus, $L$. notalensis, et s'efforcent de tirer les conclusions protiques de celte étude, notamment en ce qui concerne la lutte contre ces mollusques et l'époque la plus favorable pour traiter les animaux porteurs de fosciolo gigontico.
\end{abstract}

A la suite d'enquêtes effectuées de 1954 à 1968 chez divers rumınants et chez les équidés de la République du Tchad (GRABER, 1963), il est vite apparu que les affections parasitaires provoquées par les Trématodes du foie, de l'appareil digestif et du système veineux tenaient une place essentielle dans la pathologie des animaux domestiques vivant dans les zones comprises entre le $9^{e}$ et le $12^{e}$ parallèle.

Ces helminthes ont un cycle biologique complexe qui nécessite, à un certain moment, le passage obligatoire par un mollusque pulmoné d'eau douce basommatophore.

Devant l'importance prise par ce problème, il a été décidé, dès 1965, d'étudier de façon plus approfondie :

- les mollusques vecteurs et leur répartition géographique :

(*) Instifut d'enseignement zootechnique ef vétérinaire d'Afrique centrale. FESAC. Fort-Lamy.

(**) Instifut d'Elevage et de Médecine Vétérinaire des Pays tropicaux. Laboratoire de Farcha. Fort-Lamy.
- les cycles évolutifs des espèces parasites. dominantes (Fasciola gigantica, Paramphistamum. microbothrium, Carmyerius spatiosus) ;

- les répercussions de ces helminthiases sur la santé de l'animal ;

- les tratements antidistomiens.

Pour ce faire, il était absolument nécessaire, dans un premier temps, de créer un élevage de mollusques répondant aux trois impératifs suivants :

- être d'un prix de revient très bas.

- ne demander qu'un entretien réduit,

- permettre d'obtenir le plus grand nombre. possible d'individus.

Les conditions climatiques très dures régnant dans la région de Fort-Lamy rendaient à priori cet élevage très aléatoire.

Après bien des tâtonnements, une méthode a été mise au point fin 1966 qui a permis une série d'observations intéressantes sur les variations: 
saisonnières de certaines populations de mollusques.

\section{TREMATODES PARASITES DES RUMINANTS DOMESTIQUES ET DES ÉQUIDÉS}

Sur 12.795 animaux domestıques autopsiés au Tchad, 17 espèces différentes de Trématodes ont jusqu'c̀ présent été rencontrées (GRABER 1967), appartenant à 6 familles et à 11 genres différents (tableau no 1).
La chèvre est en général assez peu parasitée sauf par Paramphistomum microbothrium.

Le mouton comme le jeune zébu hébergent surtout des Paramphistomidae et des schistosomes. Chez les ovıns, la bilharziose intestinale entraîne chaque année des pertes, variables selon les régions, la zone la plus contaminée étant actuellement celle du Lac (Iseirom-Massakory).

Les zébus adultes $(+)$ par contre sont porteurs d'un très grand nombre de trématodes, souvent associés par 2, 3, 4 ou 5.

TABLEAU $\mathrm{N}^{\circ} \mathrm{I}$

Trématodes des animaux domestiques - Taux d'infestation (en F.100).

\begin{tabular}{|c|c|c|c|c|c|c|c|}
\hline Espèces en cause & $\begin{array}{c}\text { Jeunes } \\
\text { zébus } \\
(1567)+\end{array}$ & $\begin{array}{c}\text { Zébus } \\
\text { adultes } \\
(4743)+\end{array}$ & $\begin{array}{l}\text { Moutons } \\
(5382)+\end{array}$ & $\begin{array}{l}\text { Chèvres } \\
(781)+\end{array}$ & $\begin{array}{c}\text { Dromadaires } \\
(159)+\end{array}$ & $\left(\begin{array}{l}\text { Anes } \\
110)\end{array}\right.$ & $\begin{array}{l}\text { Chevaux } \\
(53)+\end{array}$ \\
\hline Diorocoetium hospes & - & 1,1 & 0,2 & - & - & - & - \\
\hline Fasciola gigantioa & 2,8 & 28,7 & 0,8 & 0,38 & - & 0,1 & - \\
\hline $\begin{array}{l}\text { Cotylophoron } \\
\text { coty Zophorum }\end{array}$ & 0,3 & 1,4 & $a, 7$ & 0,5 & - & - & - \\
\hline $\begin{array}{l}\text { Calicophoron } \\
\text { calicophorum }\end{array}$ & 0,06 & 0,3 & - & - & - & - & - \\
\hline $\begin{array}{l}\text { Stephomophaxynx } \\
\text { compactus }\end{array}$ & - & 1,6 & - & - & - & - & - \\
\hline Caticophoron raja & - & 0,002 & - & - & - & - & - \\
\hline Caiteophoron ijimai & - & 0,15 & - & - & - & - & - \\
\hline $\begin{array}{l}\text { Bothriophoron } \\
\text { bothriophoron }\end{array}$ & - & 0,3 & - & - & - & - & - \\
\hline $\begin{array}{l}\text { Giguntocotyle } \\
\text { symmeri }\end{array}$ & - & 0,12 & - & - & - & - & - \\
\hline $\begin{array}{l}\text { Paramphistomum } \\
\text { microbothrium }\end{array}$ & 14,1 & 24,6 & 19,9 & 13,7 & - & - & - \\
\hline Carmyexius spatiosus & 1,3 & 7,9 & 0,77 & 0,5 & - & - & - \\
\hline $\begin{array}{l}\text { Carmyerius } \\
\text { papiziatus }\end{array}$ & 0,7 & 1,7 & 0,4 & 0,1 & - & - & - \\
\hline $\begin{array}{l}\text { Canmyerius } \\
\text { parvipapizzatus }\end{array}$ & - & 0,15 & 0,05 & - & - & - & - \\
\hline Carmyerius graberi & 0,1 & 0,4 & 0,05 & - & - & - & - \\
\hline Schistasama bovis & 16 & 33,6 & 10 & 0,1 & 5 & 7,2 & 22,6 \\
\hline Sehistosoma mattheei & 0,5 & 0,6 & 0,17 & - & - & - & - \\
\hline $\begin{array}{l}\text { Gastrodiscus } \\
\text { aeguptiaaus }\end{array}$ & - & - & - & - & - & 33,6 & 56,6 \\
\hline
\end{tabular}

$+=$ Animaux autopsiés

Chez le dromadaire, le parasitisme est négligeable.

Chez les équidés, la gastrodiscose ef la schistosomiase ont une certaine importance surtout chez le cheval.
Dicrocoelium hospes se rencontre dans toute la vallée du Haut-Logone, le Nord Cameroun ef

(*) A partir de 3 ans. 
l'Ouest de la RCA. Sa présence, en zone sahélienne, n'a été signalée qu'une fors à Abéché $(+)$.

Fosciola gigantica est presque inexistante à l'Est et au Nord d'une ligne théorique joignant Mao à Am-Timam. Les bassins du Chari, du Logone et le lac Tchad constituent des foyers importants de distomatose bovine.

II en est de même pour les Paramphistomidae et les Gastrothylacidae de la panse.

Quant aux schistosomes, ils paraissent beaucoup mieux répartis et on les retrouve fréquemment très au Nord au Bahr-el-Ghazal et jusque dans l'Ennedi.

D'une façon générale, le parasitisme par trématodes hépatiques et gastriques (tableau
L'étude de ces parasıtoses présente donc un intérêt certain pour le Sud de la Républıque du Tchad où le Service de l'Agriculture cherche actueliement à développer conjointement le Coton ef l'Elevage qui, pour l'instant, est presque enłièrement limité à la culture attelée (40.000 têtes en 1967).

\section{LES MOLLUSQUES VECTEURS}

La faune malacologique a déjà fait au Tchad l'objet de nombreux travaux. Les premiers exemplaires ont été récoltés, il y a cent ans, par le Docteur Gerhard ROHLFS au cours d'une traversée du Sahara. Depuis, plusieurs missions ont permis de rassembler un important matériel :

TABLEAU $N^{0}$ II

Trêmatades du zébu adulte-Taux d'infestation par Préfecture (en p, 100).

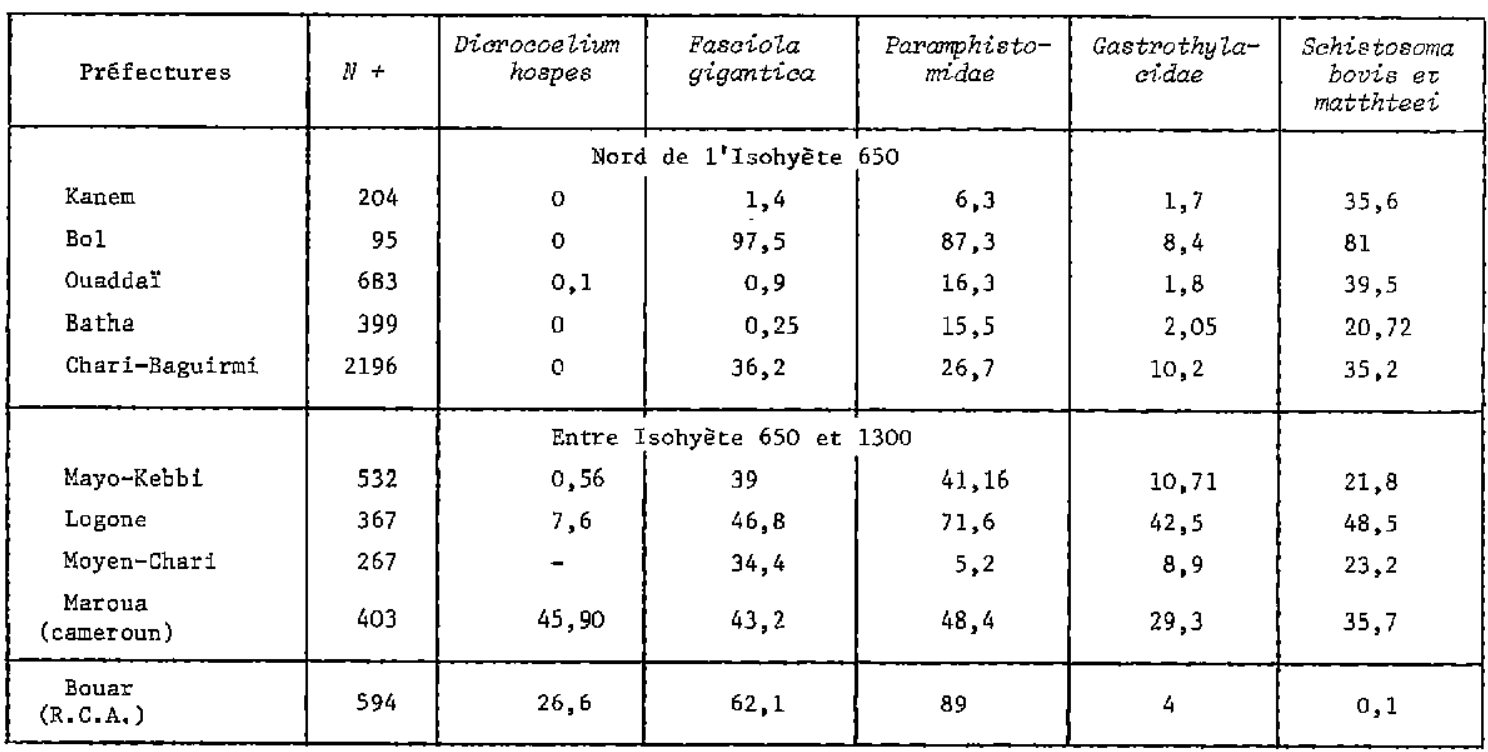

+ = Nombre d'animaux autopsiés

$n^{0}$ 2) semble être plus abondant entre les isohyètes 650 (limite Nord culture coton) et 1.300 (carte $n^{\circ} 1$ ), sauf dans la basse vallée du Chari (Fort-Lamy) et dans la préfecture de Bol où le lac Tchad et son affluent principal le Chari, représentent un milieu éminemment favorable à la pullulation des mollusques vecteurs (LEVEEQUE, 1967 a).

(*) Bétail transhumant venu du Salamat. missions MONTEIL (1892 et 99), GENTIL, FOUREAU-LAMY, LENFANT, expéditions CHADEAU (1905-06), CHEVALIER ef TILHO (19061909).

GERMAIN (1935) (*) devait attacher son nom à l'étude des mollusques du lac ef des pays bas du Tchad (Sud-Ovest et Tibesti).

$(*)$ des index bibliographiques complets figurent dans cette publication. 
CARTE $n^{\circ}$ I : ISOHYETES et LIEUX de RECOLTE des VECTEURS

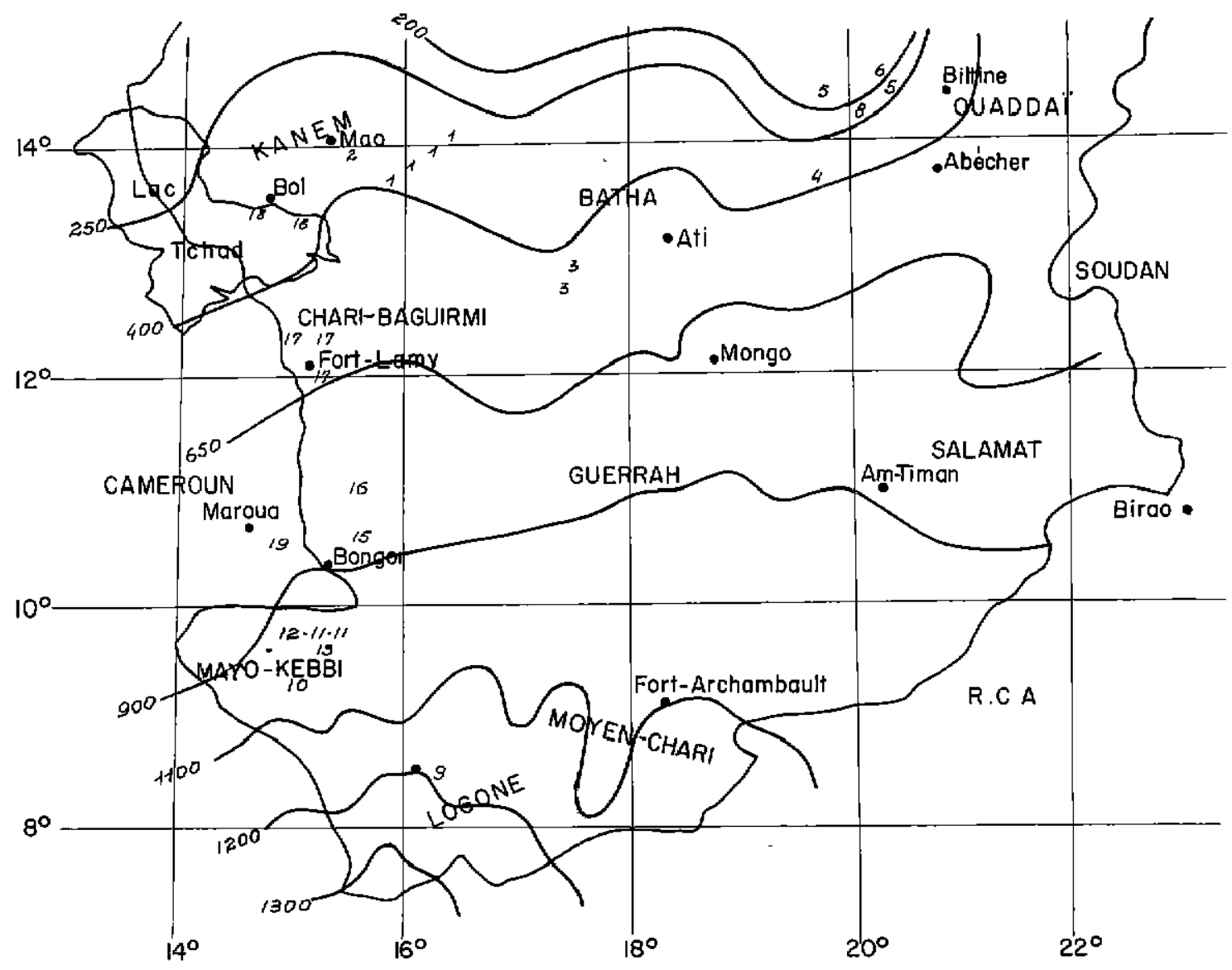

| Bahr el Ghazal, Moussoro,Imari II Loc de Fianga

2 Mao

12 Lac de Tikem

3 Lac Fitri

13 Rivière Kabia

4 Ouaddi Haddad

14 Kerchen

5 Kharmo

I5 Rivière Bo-illi

6 Kadjemeur

16 Rivière Loumia

7 Famo Ouczat Ouayali

17 Fort-Lamy

8 Arada

9 Maundou

I8 Lac Tchad(O.R.S.T.M.M)

10 Palo

19 Maroua

Plus récemment, LÉVÊQUE (1967 a) signale l'existence de 28 espèces et variétés de mollusques aquatiques dans la partie Est du lac (Est de la longitude Baga Sola).
Seules actuellement ${ }^{* *}$ ) celles qui appartiennent au groupe des gastéropodes pulmonés

(**) on ignore encore le cycle de Dicrocodium hospes. 
basommatophores ont un intérêt médical ef vétérinaire.

Ce sont $\left(^{*}\right)$ :

10 Familie des Lymnoeidoe.

Genre Lymnoeo, LAMARCK (1799).

Limnoea natalensis (KRAUSS, 1848).

$$
9-10-11-12-13-14-15-16-18-19 .
$$

20 Famille des Planorbidae.

2.1. Genre Biomphalaria, PRESTON (1910).

2.1.1. Biomphalaria pfeiffer (KRAUSS, 1848).

$$
9-10-11-12-15-18-19 .
$$

2.1.2. Biomphalario sudanica (MARTENS, 1870).

$$
11-12-18 \text {. }
$$

2.2. Genre Segmentorbis (MANDALH-BARTH, 1954).

$$
3-11-12-18 \text {. }
$$

2.3. Genre Gyroulus (CHARPENTIER, 1837).

Gyraulus costulotus (KRAUSS, 1848).

$$
9-10-11-12-17-18 \text {. }
$$

(*) les chiffres indiquent les points de récolte (voir carte $n^{0}$ 1).
2.4. Genre Anisus (STUDER, 1820).

2.4.1. Anisus notalensis (KRAUSS, 1848).

$$
9-10-11-17-18 .
$$

2.4.2. Anisus coretus (BLAINVILLE, 1826).

30 Famille des Bulidoe.

Genre Bulinus (MüLLER, 1781).

3.1. Bulinus jousseaumei (DAUTZNBERG, 1890).

$$
1-11-12-18-19
$$

3.2. Bulinus truncotus (*) rohlfsi (CLOSSIN, 1886).

$$
3-4-7-8-9-10-11-12-13-14-15-
$$
$17(* *)-18$.

3.3. Bulinus forskalı (EHRENBERG, 1831).

$1-2-3-4-5-6-7-8-9-10-11-12-13$ $-14-17-18$.

Ces pulmonés ne sont pas tous vecteurs de

\begin{tabular}{|c|c|c|}
\hline Mollusques & Parasites transmis & A ut e u $r$ \\
\hline Lymnaea natalensis & Fasciola gigantica & $\begin{array}{l}\text { Porter 1920-21; Daynes } 1967 \text {; Bitakara- } \\
\text { mire 1968; Kendal1 et Parfit } 1965 .\end{array}$ \\
\hline $\begin{array}{l}\text { Biomphaiaria } \\
\text { pfeifferi }\end{array}$ & $\begin{array}{l}\text { Schistosoma mansoni } \\
\text { Parcomphistomum sukari }\end{array}$ & $\begin{array}{l}\text { liandahl - Barth, } 1962 \\
\text { Dinnik, } 1965 \text { a }\end{array}$ \\
\hline Biomphataria sudanica & Sohistosoma mansoni. & Mendahl Barth, 1962 \\
\hline Anisus natalensis & $\begin{array}{l}\text { Carnyerius moncupatus } \\
\text { Ceylonocotyle scoliocaelium }\end{array}$ & Dinnik 1965 a \\
\hline Bulinus jousseaumei & Schistosoma curassoni & Grëtillat 1963 \\
\hline $\begin{array}{l}\text { Butinus truncatus } \\
\text { rohlf ai }\end{array}$ & $\begin{array}{l}\text { Schistosoma haematobium } \\
\text { Schistosoma curassoni } \\
\text { Schistosoma bovis } \\
\text { Earamphistomm microbothrizm }\end{array}$ & $\begin{array}{l}\text { Mandahl - Barth } 1962 \text { et } 1965 \\
\text { Grétillat I963 } \\
\text { Dinnik et Kinnik } 1965 \text { b } \\
\text { Dinnik } 1965 \text { a }\end{array}$ \\
\hline Bulinus forskali & $\begin{array}{l}\text { Schistosoma haematobium } \\
\text { Poromphistomu phillerouri } \\
\text { Stephonophorynx compactus } \\
\text { Gastrodiscus aegyptiacus } \\
\text { Schistosoma bovist }\end{array}$ & $\begin{array}{l}\text { (probablement) Mandahl -- Barth } 1965 \\
\text { Dinnlk } 1965 \text { a } \\
\text { Dinnik } 1965 \mathrm{a} \\
\text { Le Roux } 1958\end{array}$ \\
\hline
\end{tabular}
maladies parasitaires, ainsi que le montre le tableau $n^{\circ} 3$.

(*) Sans doute identique à Bulinus (Physopsie) globulosus (Franc, 1968).

(**) Mis en évidence en octobre 1968.

TABLEAU $\mathrm{N}^{\circ}$ III

Gasteropodes pulmonés d'eau douce vecteurs de maladies parasitaires

+ Fort-Lamy - Observations personnelles. 


\section{CONDITIONS CLIMATIQUES GÉNÉRALES DE LA RÉGION DE FORT-LAMY}

La région de Fort-Lamy est soumise d̀ un climat de type sahélien comportant une saison sèche et une saison humide, cette dernière s'étendant de la fin mai à octobre.

Les précipitations dont la valeur moyenne est de $578 \mathrm{~mm}$ par an pour les années 1965-1968 (tableau no 4) ont lieu en majeure partie en juillet et en août.

Le degré hygrométrique subit le contrecoup de cette situation : les pourcentages les plus bas sont relevés de décembre à mai ; de juillet à octobre its oscillent entre 80 et 90 p. 100.

Quant à la température, elle est caractérisée par des écarts journaliers importants atteignant $17-19^{\circ} \mathrm{C}$ de février à mai. A l'ombre, en avrilmal, certaınes années, les maximums dépassent $40-41^{\circ} \mathrm{C}$.

De telles conditions, très sévères, risquent de gêner les élevages : en effet, la température de l'ecu où sont disposés les moliusques dolt être supérieure à $+20^{\circ} \mathrm{C}$ (KENDALL et PARFIT, $1965)$ sans dépasser $+28-30^{\circ} \mathrm{C}$ (CLAUGHER, 1960).

Généralement, on maintient l'eau des bacs à $26^{\circ}-28^{\circ} \mathrm{C}$ (BITAKARAMIZE, 1968, DAYNES, $1967)$.

\section{MÉTHODES D'ÉLEVAGE}

De nombreuses méthodes ont été décrites selon les régıons et les latıtudes : bacs à circula-

TABLEAU $\mathrm{N}^{\bullet}$ IV

Température, humidité et pluies - Fort-Lamy (décembre 1966 à Juin 1968)

\begin{tabular}{|c|c|c|c|c|c|c|}
\hline \multirow{3}{*}{\multicolumn{2}{|c|}{ Décembre 1966}} & \multicolumn{2}{|c|}{ Humidité en p.100 } & \multirow{2}{*}{$\begin{array}{l}\text { Température } \\
\text { moyenne } \\
\text { minimale }\end{array}$} & \multirow{2}{*}{$\begin{array}{l}\text { Température } \\
\text { moyerne } \\
\text { maximale }\end{array}$} & \multirow{2}{*}{$\begin{array}{l}\text { Plules en } \\
\text { dixtèmes } \\
\text { de min }\end{array}$} \\
\hline & & $6 \mathrm{~h}$ & $12 \mathrm{~h}$ & & & \\
\hline & & 44 & 14 & 14,5 & 34,1 & \\
\hline Janvier & 1967 & 45 & 18 & 12,6 & 32,1 & \\
\hline Février & 1967 & 34 & 13 & 16,8 & 36,7 & \\
\hline Mars & 1967 & 25 & 11 & 20 & 38 & \\
\hline Avril & 1967 & 41 & 16 & 23,2 & 42 & 34,5 \\
\hline Ma1 & 1967 & 49 & 19 & 25,4 & 42 & 0,3 \\
\hline Juin & 1967 & 70 & 37 & 24,4 & 38 & 43,5 \\
\hline Juillet & 1967 & 86 & 58 & 23 & 33,4 & 170,5 \\
\hline Août & 1967 & 92 & 70 & 21,9 & 30,4 & 287,9 \\
\hline Septembre & e 1967 & 92 & 62 & 22,2 & 32,2 & 100,0 \\
\hline Octobre & 1967 & 78 & 31 & 20,6 & 36,6 & 9,9 \\
\hline Novembre & 1967 & 59 & 17 & 16,2 & 35,8 & \\
\hline Dêcembre & 1967 & 53 & 16 & 15,2 & 36 & \\
\hline Janvier & 1968 & 71 & 15 & 13,3 & 32,7 & \\
\hline Févriet & 1968 & 55 & 11 & 15,4 & 36,4 & \\
\hline Mars & 1968 & 49 & 8 & 20,2 & 39,8 & \\
\hline Avril & 1968 & 58 & 21 & 23,2 & 39,9 & traces \\
\hline Mai & 1968 & 72 & 23,5 & 24,3 & 39,1 & 37,5 \\
\hline Juin & 1968 & 8,9 & 4,3 & 22,9 & 34,4 & 115,7 \\
\hline
\end{tabular}


tion d'eau continue disposés dans une pièce où la température ne descend jamais au-dessous de $25^{\circ} \mathrm{C}$ (SWART et REINECKE, 1962), aquariums, cuves (KENDALL et PARFIT, 1965), bassins de 650 litres placés en série dans un bâtiment aéré naturellement (GRETILLAT, 1960).

Les méthodes d'élevage ont été d'ailleurs codifiées par CLAUGHER (1960).

Ces techniques ont leurs avantages et leurs inconvénients. Dans les pays où la température est élevée à certaines époques de l'année, elles nécessitent l'installation d'une pièce climatisée, ce qui augmente considérablement les coûts de recherches et crée des sujétions quelquefois importantes (surveıllance constante, personnel spécialisé, etc.).

Aussi s'est-on efforcé au laboratorre de FAR$\mathrm{CHA}$ de mettre au point des élevages simples et faciles à maintenir.

\section{Au laboratoire même.}

1.1. Des cristallisoirs de $19 \times 8 \mathrm{~cm}$, d'une capacité d'environ 2,5 litres, ont été remplis d'eau claire et de végétaux aquatıques, essentiellement Ceratophyllum demersum. Its ont été placés dans un couloir aéré et constamment balayé par un fort vent soufflant du Nord. Ils ont reçu divers mollusques recueillis dans des mares temporares situées à la périphérie immédiate de Farcha.

La nourriture était à base de salade ef l'eau provenait du forage du laboratoire.

A partır de mars 1965, la température ambiante est montée jusqu'à 33-350. Il a fallu alors entourer les cuves de linges mouillés pour obtenir une eau relativement fraîche, ne dépassant pas $30^{\circ} \mathrm{C}$.

Cette méthode a été abandonnée, car :

- Il est nécessaire de vidanger les bacs deux ou trois fois par semaine. Le siphonnage qui accompagne cette opération entraîne à l'extérieur un grand nombre de jeunes qui sont perdus pour l'élevage :

- à cette époque de l'année, de violents vents de sable apparaissent, couvrant l'eau des cristallısorrs d'un véritable voile qui intoxique les mollusques,

- si Bulinus forskali se développe à peu près normalement, il n'en n'est pas de même pour Limnaed natalensis, Bulinus truncolus, Bulinus jousseaumei et Biomphaloria pfeifferi qui n'arrivent pas à se multiplier convenablement. II en résulte un élevage précaire qui ne répond ni aux buts fixés, ni aux besoins.

1.2. D'autres dispositions ont alors été prises. Des observations faites en divers points de récolte (Fort-Lamy, Lac Tchad, Lac Fitrı, Lac de Fianga) ont montré que ces gastéropodes pulmonés vivent en général dans des eaux très chargées en matières organiques dont le $\mathrm{pH}$ varie de 7,6 à 8,6 , alors que l'eau du forage du laboratoire a un $\mathrm{pH}$ de 6,6 (tableau no 5).

Pour serrer les conditions naturelles de plus près, des bacs plus grands $(18 \times 30 \mathrm{~cm})$ en matıère plastique, d'une capacité de 10 litres environ, ont alors été utilisés et disposés de la même façon que dans l'expérience précédente.

On dépose dans le fond de chacun d'eux de la boue prélevée dans les marigots proches du laboratoire que l'on mélange avec de l'eau claire.

On laisse déconter une quinzaine de jours, puis on ajoute des Cerotophyllum et des mollusques qui sont nourris avec des feuilles de salade.

On complète, au fur et à mesure de son évaporation, l'eau des bacs dont la vidange est faite toutes les trois semaines seulement.

La température se maintient entre 27 et $28^{\circ} \mathrm{C}$ entre septembre et novembre et entre 23 et $26^{\circ} \mathrm{C}$ entre novembre et janvier.

Par rapport à la précédente, cette méthode permet une meilleure croissance de $B$. jousseaumi, $B$. truncatus et $L$. natalensis. Les pertes de jeunes, au moment du siphonnage et des vidanges, sont réduites au minimum.

Cependant la prolifération de troıs principales espèces est telle que le nombre de récipients susceptıbles de les recevoır dolt être considérablement augmenté, ce qui demande beaucoup de place et de manipulations, d'où des pertes de temps appréciables.

De plus, à l'intérieur des bacs, la concurrence vitale joue au profit des adultes. Les jeunes mollusques, les plus aptes à l'infestation expérimentale par Fosciolo ou Paramphistomum ne sont pas assez nombreux et c'est là le prıncipal défout de la méthode.

II est bon de noter également que Bulinus forskali, dans ces conditions, ne se multiplie pas.

Ceffe technique a néanmoins été conservée au laboratoire pour des essais limités à quelques 


\begin{tabular}{|c|c|}
\hline $\begin{array}{r}\text { Résistiv } \\
\mathrm{pl}\end{array}$ & $\begin{aligned} \therefore C & =8,423 \\
C & =6,6\end{aligned}$ \\
\hline Cations : & j̃egrês \\
\hline $\mathrm{Ca}^{++}$ & 4 \\
\hline $\mathrm{Mg}^{++}$ & 2 \\
\hline $\mathrm{Na}^{+}, \mathrm{K}^{+}$ & 4 \\
\hline $\mathrm{Fe}^{+++}$ & - \\
\hline $\mathrm{H}^{+}$(acides forts) & 0 \\
\hline Total des cations & $\overline{10}$ \\
\hline Anions & Degrës \\
\hline Bicarbonates $\mathrm{CO}_{3} \mathrm{H}^{-}$ & 7 \\
\hline Carbonates $\quad \mathrm{CO}_{3}^{--}$ & 0 \\
\hline Sulfates & 2 \\
\hline Chlorures $\mathrm{Cl}^{-}$ & 0 \\
\hline Silicates & 1 \\
\hline Oxydriles $\mathrm{OH}^{-}$ & 0 \\
\hline Tota1 des antons & 10 \\
\hline
\end{tabular}

\begin{tabular}{cr} 
Mililéquivalents & $\mathrm{mg} / 1$ \\
0,8 & 16 \\
0,4 & 5 \\
0,8 & 18 \\
- & - \\
0 & 0 \\
\hline 2 & 39 \\
Miliéquivalents & $\mathrm{mg} / 1$ \\
1,4 & 85 \\
0 & 0 \\
0,4 & 19 \\
0 & 0 \\
0,2 & 8 \\
0 & 0 \\
2 & 112
\end{tabular}

Salinité totale $=151 \mathrm{mg} /$ litre.

mois d'hiver et n'intéressant qu'un petit nombre d'individus des genres Gyraulus et Anisus.

\section{$2^{\circ}$ A l'extérieur du laboratoire sous abri.}

Fort des enseignements tırés de cette expérience, Il a alors été décıdé de recréer le plus exactement possıble les facteurs écologiques qui contribuent dans la nature à l'épanoulssement de la faune malacologique. Les mollusques ne sont plus élevés à f'intérieur, mais à l'extérieur du laboratoire.

De grands bacs de fer, semi-circulaires, de $3 \mathrm{~m}$ de long, $80 \mathrm{~cm}$ de large et $40 \mathrm{~cm}$ de haut, d'une contenance de 750 litres, ont été installés au ras du sol contre le mur d'un bâtiment. Un auvent de 2,10 m les abrite du soleil. L'orientation est Nord Nard-Est, ce qui les met sous vents domınants du Nord-Est (novembre à avril) ou de l'Ovest Nord-Ouest (avrll à octobre).

Cette disposition entraîne un certain brassage des eaux de surface, empêche la formation de voiles bactériens, et maintient une température convenable dans chaque bac. La luminosité est suffisante pour le développement de la flore et la microflore, le soleil ne frappant les bacs que le matin ( 1 h) et l'après-mıdi ( 1 h).

Comme dans le cas précédent, le fond des récipients est recouvert de boue arrachée aux mares de volsinage. On procède à la mise en eau. Un mois est nécessare pour que la boue se décante et qu'une fine pellicule de rovilie apparalsse sur les parois.

On ensemence alors avec :

- des Ceratophyllum qui permettent une meilieure oxygénation des eaux :

- des Nymphea rufescens dont les fevilles sont les lieux de ponte préférés des mollusques :

- des Oscillatoria fournis par la bove d'origine et qui se fixent sur les surfaces inférieures des bacs, une fors la couche de roville formée.

Tous les dix jours environ, on rajoute un certain volume d'eau, variable selon la saison, destiné à compenser l'évaporation et à maintenir le niveau à $5 \mathrm{~cm}$ des bords. Aucun bac n'a été vidé depuis octobre 1965.

La protection des mollusques et de leurs pontes contre divers prédateurs (marabouts, hérons, 
crapauds) est réalisée au moyen de grillages moustiquaires.

Des fevilles de salades sont régulièrement distribuées : elles assurent la nourriture des mollusques adultes, les jeunes sé contentant des algues (Oscillatoria) très abondantes dans le nouveau milieu.

II n'est pas possible d'obtenir Immédiatement une population importante et homogène dans chaque récipient. Un temps de latence de 2 à 3 mols est nécessaire. II faut attendre en effet que le $\mathrm{pH}$ acide de départ qui entrave la multiplication des pulmonés passe à 7,6-8,3, ce qui se produit progressivement sous l'effet conjugué de la bove et des micro-organısmes mêlés à l'eau du forage.

Le tableau no 6 montre les changements survenus dans la composition chimique de l'eau des bacs, un an après leur mise en service (cf. tableau $n^{\circ} 4$ ). En définitive, expérimentalement, on obtient peu d̀ peu des equx comparables à celles du Nord du lac Tchad où Bulinus, Limnées et Bıomphalaria sont très fréquemment rencontrés.
TABLEAU $\mathrm{N}^{\circ} \mathrm{VI}$

Analyse chimique de l'eau des bacs après un an de mise en service (Dëc. 1967).

\begin{tabular}{|c|c|c|c|c|c|}
\hline Bacs & $\begin{array}{l}\text { Rêsistivité } \\
\text { à } 25^{\circ} \text { en } \\
\omega / \mathrm{cm}\end{array}$ & $\begin{array}{l}\mathrm{pH} \text { à } \\
25^{\circ} \mathrm{C}\end{array}$ & $\begin{array}{l}\mathrm{HCO}_{3}^{-} \\
\mathrm{meq} / 1\end{array}$ & $\begin{array}{c}\mathrm{Co}_{3}^{-} \\
\text {meq/1 }\end{array}$ & $\begin{array}{c}\mathrm{Cl}^{-} \\
\text {meq } / 1\end{array}$ \\
\hline A & 476 & 7,6 & 4,95 & 0 & 0 \\
\hline B & 370 & 8,3 & 3,10 & 0,80 & 0,1 \\
\hline$c$ & 353,7 & 8,3 & 3,40 & 0,30 & 0 \\
\hline D & 533 & 7,6 & 5,25 & 0 & 0,3 \\
\hline
\end{tabular}

Par ailleurs, des relevés thermiques régulıers effectués tous les jours vers 17 heures du 14 octobre 1967 au 15 juin 1968 indiquent que la température maximale des bacs n'a jamals dépassé + $27,5^{\circ} \mathrm{C}$, même à l'époque la plus chaude de l'année (avril-mai) et que la température minımale oscille entre $16^{\circ} \mathrm{C}$ et $17,5^{\circ} \mathrm{C}$ en décembre ef en janvier (graphique no 1 ).

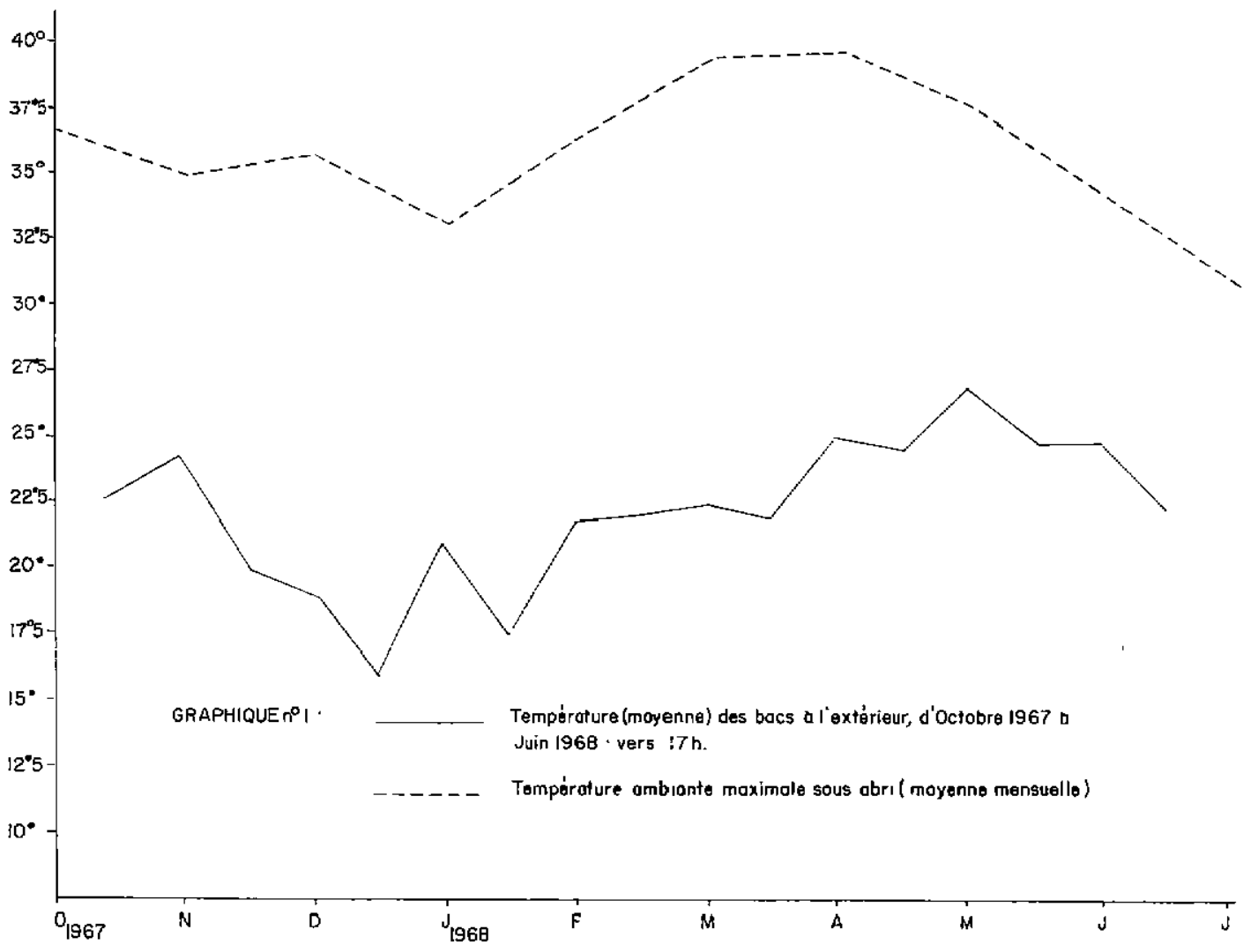


La population des bacs est très largement suffisante pour des études de population et pour des essais d'infestation. En décembre 1968, il a été dénombré dans les bacs 1 et 4 de 1.300 à 1.430 jeunes $B$. truncatus de 3 à $4 \mathrm{~mm}$ et, dans les bacs 2 et 3 , de 800 à 1.300 L. natolensis adultes de 7 à $14 \mathrm{~mm}$.

\section{RÉSULTATS}

Il ne sera question $\mathrm{i} C$ q que des quatre espèces principales : B. forskalı, Bulinus truncatus, Bulinus jousseaumei et $L$. notolensis.

\section{$1^{0}$ Au laboratoire.}

1.1. Bulinus forskali (graphique no 2).

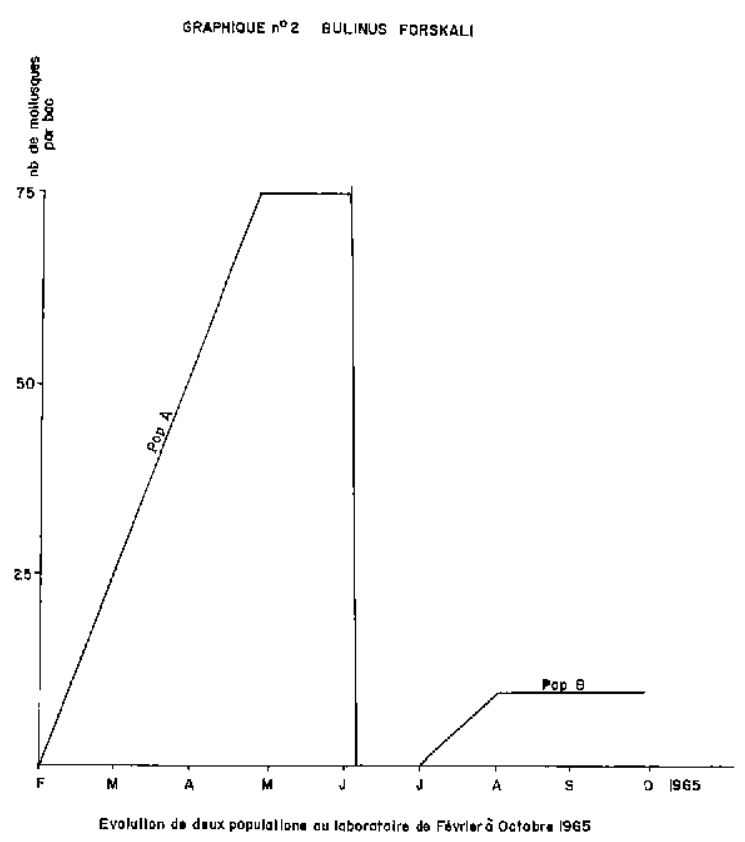

Ce bulin se reproduit de façon satisfaisante à la fin de la salson sèche de mars à juin. A partir de juillet l'élevage périclite et se maintient péniblement à un niveau très bas durant toute la saison des pluies (juillet à octobre).

Par ailleurs, B. forskali supporte très mal la concurrence des autres mollusques : lorsque, dans un bac, coexistent $B$. truncatus, $B$. natalensis et $B$. forskali, ce dernier est automatiquement éliminé. C'est ce qui se passe également dans la nature : les mares autour de Fort-Lamy ont des populations homogènes très importantes de $B$. forskall, seule espèce recueillie jusqu'en octobre 1968. Par contre, sur les rives du lac de
Fianga où souvent plusieurs genres cohabitent (B. truncatus, B. jousseaumei, L. notalensis, B. pfeifferi) on ne trouve que quelques individus isolés. Les rares colonies de $B$. forskali sont farbles et strictement localisées à des zones dont les autres pulmonés sont absents.

1.2. Bulinus truncatus, Bulinus jousseaumer et Limnoea notolensis.

Dans les bacs extérieurs, comme au laboratoire, on assiste, au cours des années 1966 et 1967, d̀ un accroissement massif des populations à partir du mois d'octobre. Le maximum est atteint entre novembre et février (graphique $n^{\circ}$ 3). A compter de mars-avril, le nombre de mollusques baisse considérablement. La chute est brutale et, durant l'hivernage, il ne subsiste que quelques adultes, ce que confirment d'autres études effectuées de mars 1965 à juin 1967 : des bulins recueillis en juin 1965 au lac Fitri et des limnées provenant du lac Tchad ont végété, et n'ont commencé à se reproduire de façon normale que vers le 15 octobre.

Là encore, lorsque B. truncatus, B. jousseaumei et $L$. natalensis sont présents dans un même bac, un phénomène de concurrence vitale apparaît au détriment des bulins (graphique $n^{\circ} 4$ ) qui survivent d'ailleurs en partie. II est possible que le milieu artificiellement créé dan's les bacs jove un certain rôle, car l'eau qui y est contenue est claire, non brassée, ce qui convient bien aux limnées, mass non aux bulıns qui préfèrent les eaux peu profondes, stagnantes et très chargées en matıères organiques.

Quant aux Biomphalario, ils ne tolèrent pas d'être mis en contact avec d'autres gastéropodes. Dans ce cas, la croissance s'orrête rapidement, bien que le milieu dans lequel ils se trouvent ne diffère pas fondamentalement de leur milieu d'origine.

Que ce soit pour B. forskali, B. truncatus, B. jousseaumei, $L$. notalensis ou Biomphalaria sp., on a toujours intérêt à isoler les espèces et à les mettre dans des bacs séparés.

\section{$2^{\circ}$ Concordance des résultats obtenus au labo- ratoire et des observations faites sur le terrain.}

\subsection{Bulinus forskali.}

Dans les mares autour de Fort-Lamy, le bulin se développe au moment des premières pluies 
GRAPHIQUE $n^{\circ} 3$ : Ełude comparée de deux populations de mallusques (Septembre1965 a

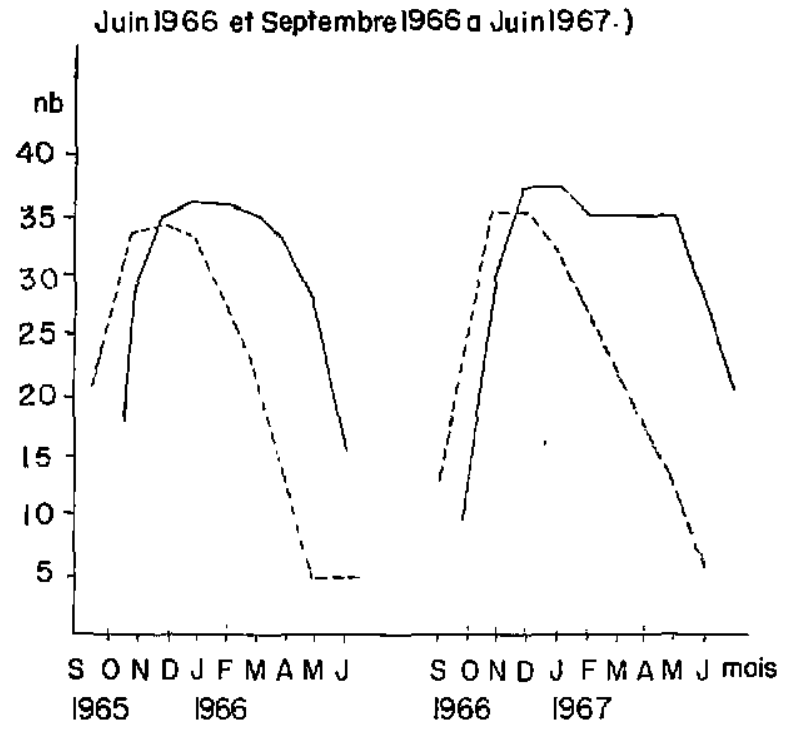

GRAPHIQUE n ${ }^{\circ} 4$ : Concurrence dans un même boc entre diverses populations de

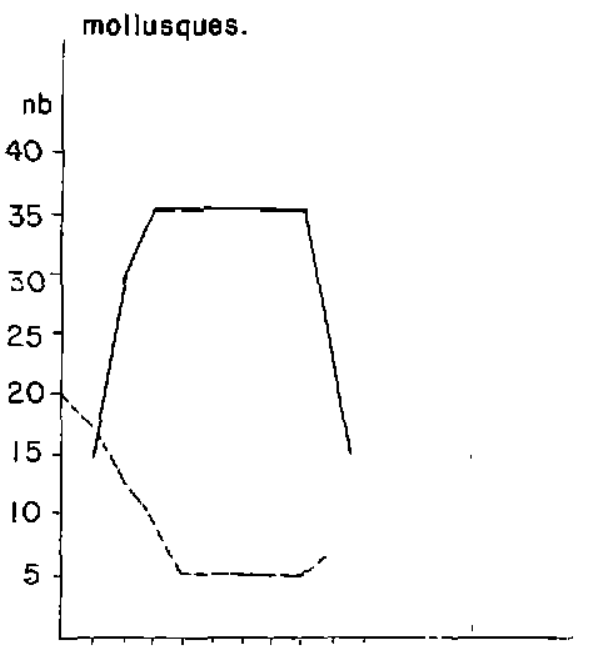

SONDJFMAMJJ mois

$1967 \quad 1968$

Limnoeo natalensis

Bulinus truncatus rohlfsi

Nombre de mollusques collectés sur une feuille de laitue en 30 secondes

qui aboutıssent à la mise en eau des marigots. Leur population croît rapidement de juillet à septembre, puis cesse d'augmenter dès la $15^{\mathrm{e}}$ semaine (LÉVÊQUE, 1967 a) avec production d'individus de faible taille qui vont au moment de l'assèchement des collections d'eau (début décembre) passer la saison sèche enfouis dans le sol jusqu'à la saison des pluies suivante.

Pour B. forskali, il y a donc distorsion entre le cycle expérimental au laboratoire et le cycle naturel. Le même phénomène a été signalé ou Khouzistan pour Bulinus truncotus par GAUD ef Coll. (1962). Aucune explication valable ne peut être actuellement fournie.

2.2. Bulinus truncatus, Bulinus jousseaumei et Limnaea natalensis.

Le cycle biologique naturel et le cycle expérimental semblent se recouper assez étroitement. En effet, des récoltes effectuées en février-mars en cing points différents du lac de Fianga par dix personnes travaillant en moyenne 4 heures dans la matinée, ont permis de dénombrer un grand nombre de mollusques (tableau no 7 ). Deux mois et demi plus tard à la fin mai, les populations diminuent sensiblement, sans que l'on puisse vraiment rendre responsable de cet état de chose la baisse saisonnıère du niveau du Lac ou son réchauffement en avril-mai, puisqu'au laboratoire le même phénomène est observé à la même époque dans des bacs où la température ne dépasse pas $27.5^{\circ} \mathrm{C}$.

Au lieu dit Folmaye, les fluctuations sont encore plus nettes:

\begin{tabular}{|c|c|c|c|}
\hline Espèces & $\begin{array}{c}\text { Février- } \\
\text { mars } \\
1967\end{array}$ & $\begin{array}{c}\text { Fin maı } \\
1967\end{array}$ & $\begin{array}{l}\text { Dimi- } \\
\text { nution }\end{array}$ \\
\hline - & - & - & - \\
\hline $\begin{array}{l}\text { Bulinus jousseoumei } \\
\text { Bulinus fruncatus }\end{array}$ & 1.327 & 108 & $-91,9$ p. 100 \\
\hline Limnaea notolensis & 86 & 12 & $-86,1$ p. 100 \\
\hline
\end{tabular}

\section{APPLICATIONS PRATIQUES}

Elles sont, au Tchad, de deux ordres :

10 En matière de destruction des gastéropodes vecteurs par des molluscicides, il est nécessaire de tenir compte des cycles saisonniers.

Pour Bulinus forskali en zone sahélienne, l'intervention devra avoir lieu à la fin de la saison des pluies en octobre, quand le volume des mares se réduit et avant que les mollusques 
TABLEALI N ${ }^{\circ}$ VII

Evolution des populations de mollusques - Lac de aianga.

\begin{tabular}{|c|c|c|c|c|}
\hline Es p èce & $\begin{array}{c}\text { Févier-Mars } \\
1967 \\
\text { total }=6099\end{array}$ & $\begin{array}{l}\text { Fin Mai } 1967 \\
\text { total = } 1421\end{array}$ & \multicolumn{2}{|c|}{$\begin{array}{c}\text { Diminution } \\
\text { ou } \\
\text { Augmentation }\end{array}$} \\
\hline $\begin{array}{l}\text { Butinus truncatus } \\
\text { Butinus jousseaumei }\end{array}$ & 5516 & 913 & \multicolumn{2}{|c|}{$-83,5$ p. 100} \\
\hline Butinus forskali & 197 & 27 & $-87,3$ & $"$ \\
\hline Lumaraea natazensis & 244 & 106 & $-57,6$ & $"$ \\
\hline Eiomphatamia pfeifferi + & 56 & 102 & +45 & $"$ \\
\hline Biomphataria suadrioa + & 53 & 66 & +20 & $"$ \\
\hline Segrentombte congustua + & 33 & 207 & $+B 4$ & $"$ \\
\hline
\end{tabular}

$+=$ Ces trois mollusques vivent habituellement dans les racines flottantes des graminaes et sur les

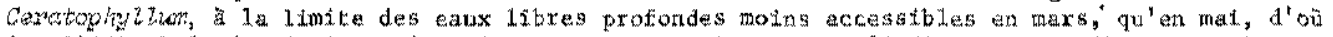
des deffícultếs de récolte quí expliquent pourquoi le noubre d'indtvidus recueillïs est plus important à la fin de la saison sèche.

n'entrent en «dormance ». Ces opérations sont parfoitement réalisables lorsqu'il s'ogit de mares temporaires sans poisson, sèches en décembre-janvier.

Pour Bulinus jousseoumei, Bulinus truncotus et Limnaea natalensis, sur les bords des rivières et des lacs, l'épandage de produits anti-mollusques se fera à l'époque des basses eaux, quand les populations ont tendance à régresser (d'avril à juin). On a ainsi toute chance d'éliminer les aduites, les jeunes et, si possible, les pontes. $2^{\circ}$ En ce qui concerne fasciola gigantica, le vecteur Limnaea natolensis recommence à foisonner en zone soudanienne da partir de novembre. époque où les animaux quittent les points hauts pour aller pâturer dans des bas-fónds marécageux riches en vecteurs.

Dans les conditions du Tchad, à partir d'un cuf de Fasciola gigantica, le stade de métacercaires infestantes lıbres dans la nature est atteint au bout de 3 mois.

On peut donc considérer que l'Infestation des

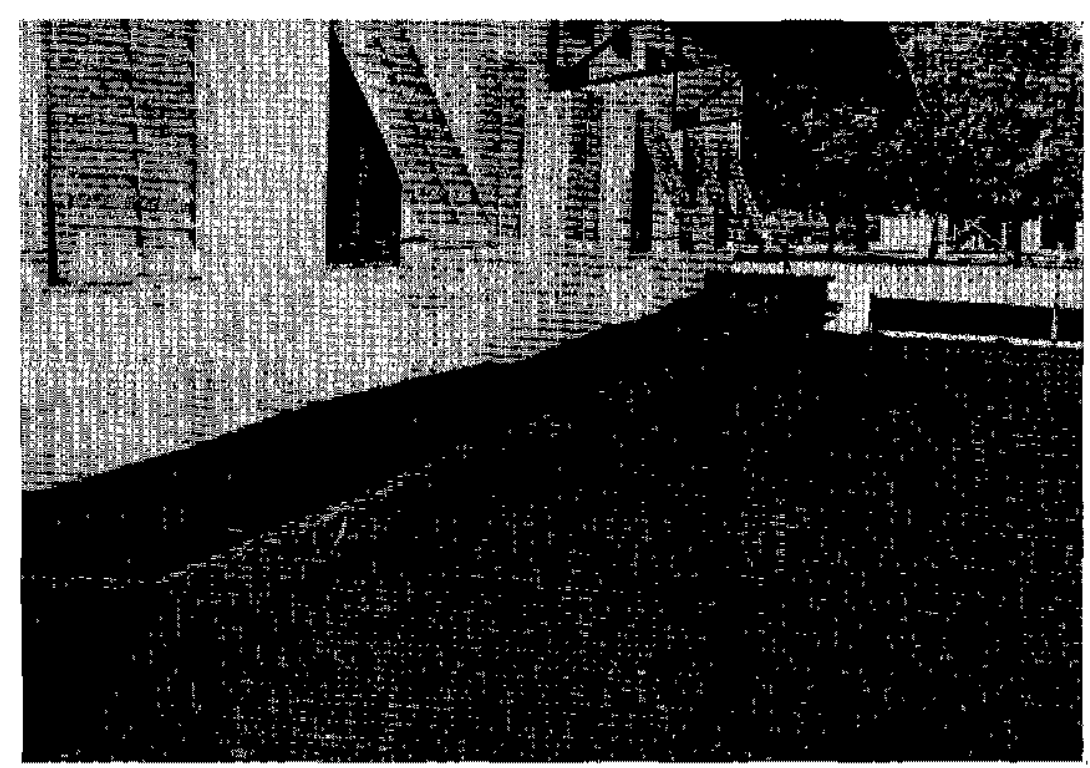

Photo 1. - Batterie de bocs. 


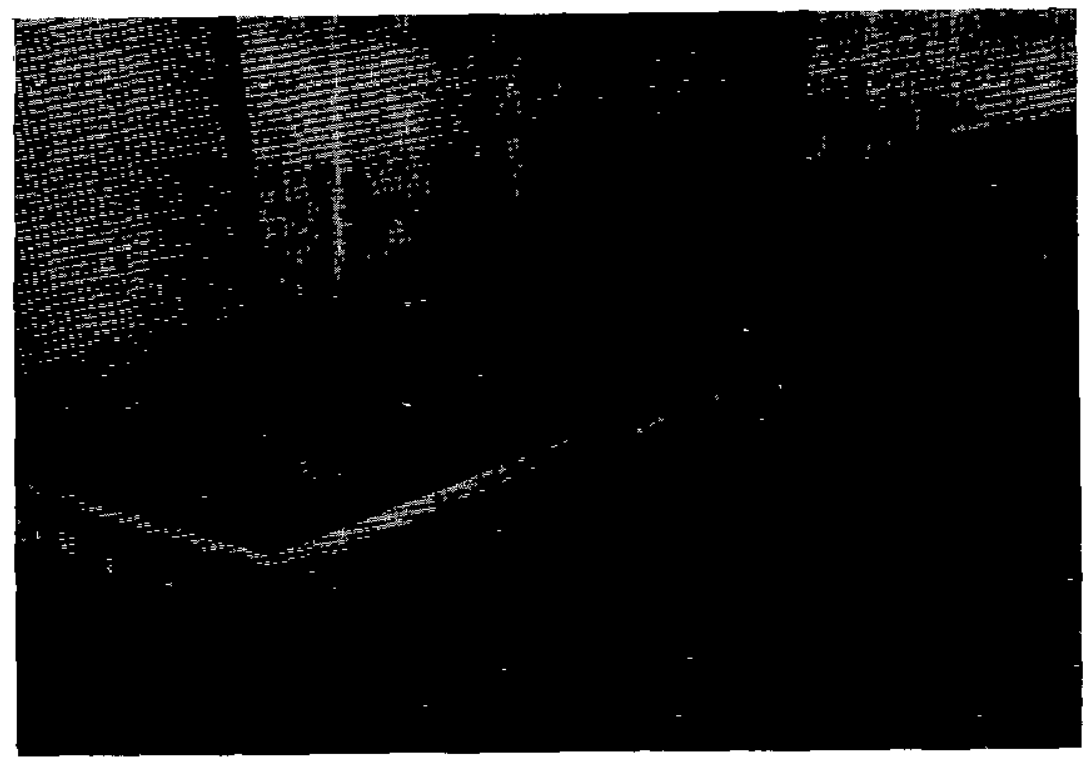

Photo 2. - Bac ensemencé.

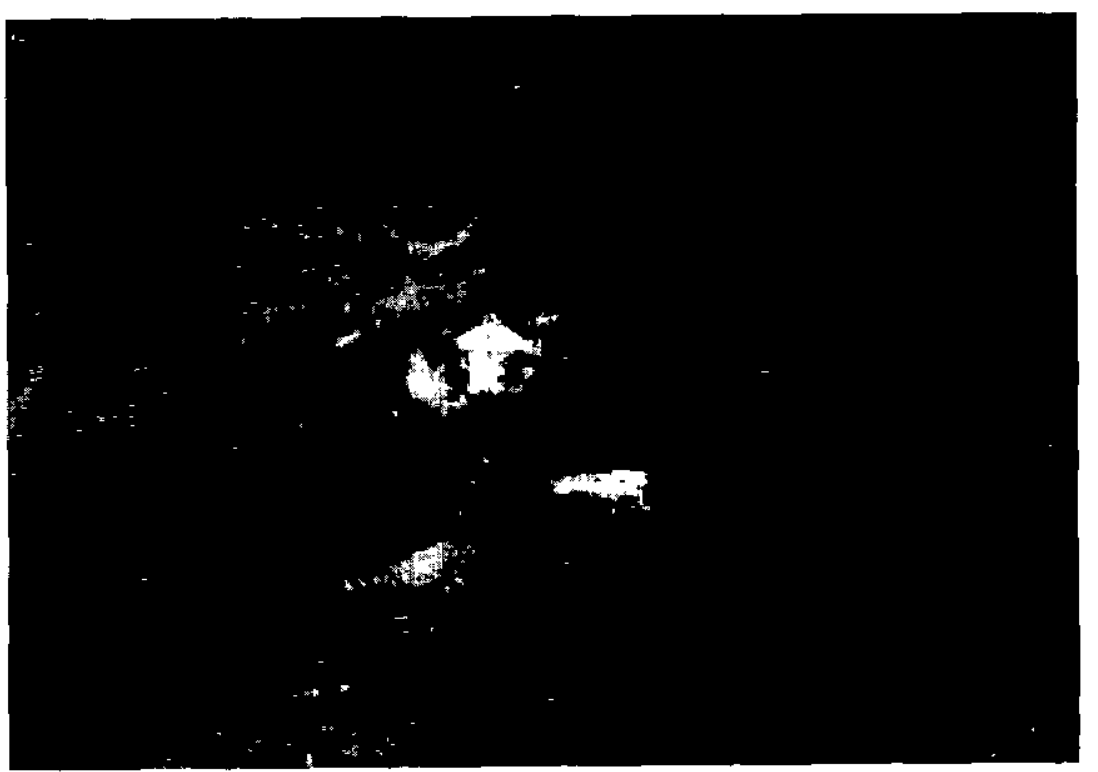

Photo 3. - Limnées sur une feuille de salade. 
animaux domestiques est susceptible de se réaliser dès février de l'année suivante. Elle se poursuivra jusqu'à la fin mai. Au-delà, la diminution saisonnière des populations de Limnées et la réapparition aux premières pluies des mares de surface où le bétail va boire et dont ces gastéropodes sont absents rendent plus difficile la contamination du bétail.

Il faut environ 136 jours (*) pour que la métacercaire absorbée par l'anımal donne un Distome adulte môr, capable de pondre des œufs décelables dans les selles.

Dans le Sud et l'Ouest du Tchad, la Distomatose bovine est une maladie de fin de saison sèche début de saison des pluies (15 mai-15 aoùt), ce que confirment les observations faites tant d̀ l'abattoir que sur le terraın (GRABER, 1967).

Ces notions ont une très grande importance en matière de lutte antidistomıenne : les traitements systématıques des anımaux de culture attelée seront effectués en mai-juın, quand les Trématodes presque mûrs peuvent être facilement touchés et détruits par les anthelminthiques actuellement disponibles.

Au Tchad, dans un premier temps et pour plus de sécurité, on recommande un second traltement «préventif» en octobre-novembre, de manière à ce que les animaux, quand ils iront dans les pâturages de saison sèche, n'hébergent plus de Fasciola.

\section{CONCLUSIONS}

$1^{0}$ Les affections parasitaires dues à des Trématodes hépatıques, stomacaux ef veıneux (Fasciola, Paramphistomum, Carmyerius et Schistosoma) ont une grande importance dans la pathologie du bétail adulte des zones Sud et NordOuest de la République du Tchad.

Le cycle évolutif de ces helmınthes nécessite

(*) BIRGI et GRABER. Non publié. la présence de mollusques pulmonés d'eau douce appartenant aux genres Lymnoea et Bulinus.

$2^{\circ}$ Compte tenu des conditions climatiques sévères de la régıon de Fort-Lamy, l'élevage de mollusques au laboratoire dans des pièces non clımatisées n'est réalısable qu'à une certaine période de l'année.

Pour pallier cette difficulté, les mollusques ont été disposés à l'extérieur, dans des bacs de 750 litres protégés par un auvent et orientés Nord-Nord-Est sous vents dominants. Les résultats sont favorables et aboutissent à la production d'un très grand nombre d'individus.

30 Bulinus forskali, fréquemment rencontré dans les mares du Sahel a un cycle saisonnier qui suit étroitement la saison des pluies et la mise en eau des collections d'eau (de juillet à décembre).

Bulinus Jousseaumei, Bulinus truncatus, et limnaea natalensis pullulent de novembre à mars le long des rivières et des lacs. Par contre, de mai à octobre, la population se mantient à un niveau très bas.

40 Dans les bacs d'élevage, chaque espèce doit être soigneusement séparée car apparaissent des phénomènes de concurrence qui amènent très rapidement la disparıtion de certaines d'entre elles.

50 Les applications pratiques de cette étude sont envisagées sous l'angle lutte anti-mollusques et époque de traitement des animaux atteints de Distomatose hépatique à Fosciola gigantica.

\section{REMERCIEMENTS}

Les auteurs tiennent à remercier Monsieur le Docteur Mandahl-Barth et Monsieur le Professeur Fraga de Azevedo qui ont eu l'amabilité de procéder à la détermınation d'un certain nombre d'espèces, ainsı que Messieurs Queval du laboratoire de Farcha et Lemoalle de I'ORSTOM de Fort-Lamy qui ont effectué les analyses d'eau.

\section{SUMMARY}

Basommatophores fresh water pulmonate molluses, vectors of livestock parasitic diseases in Chad. Breeding possibilities in laboratory

The authors note the importance of parasitic diseases caused by some venous, gastric, hepatic tremaloda (Fasciola, Poramphistomum, Carmyerius and Schistosoma) for the adult livestock of sahelo-sudanian zones of Chad. They describe a single 
and practical method allowing the breeding of principal vectors molluses in very hard climatic conditions.

Some indications about the seasonal cycle of B. forskali, B. jousseaumei, $B$. truncatus, $L$. natalensis are furnished. The practical conclusions from this study are drawn, particularly concerning the molluscs prevention and the most propitious period to cure the animals infested by Fosciola gigantica.

\section{RESUMEN}

Moluscos pulmonados basomatoforos de agua dulce, vectores de enfermedades parasitarias del ganado en Chad. Posibilidades de cria en el laboratorio

Después de haber demostrado la importancia, para el ganado adulto de las zonas sahelo-sudanesas del Chad, de enfermedades parasitarias causadas por tremátodos hepátıcos estomacales y venosos (Fasciola, Poromphistomum, Cormyerius y esquistosomos), los autores describen un método simple y práctico permitiendo criar los principales moluscos vectores, en condiciones climáticas muy desfavorables.

Dan tambien algunas indicaciones concerniente al ciclo de temporada de B. forskal, B. jousseaumei, B. truncotus, L. notalensis. Mostran las conclusiones prácticas de este estudio, particularmente en lo concerniente la lucha contra dichos moluscos y el periodo más favorable para tratar los animales portadores de Fasciola gigantica.

\section{BIBLIOGRAPHIE}

1. ANON. - Study group on the ecology of intermediate hosts of bilharziasis. Tech. Rep. Who, 1957, no 120.

2. BITAKARAMIRE (P. K.). - Lymnaea naiolensis labory culture and production of Fasciola gigantica mefacercariae. Parasitology, $1968,58,3,653-56$.

3. CLAUGHER (D.). - The transport and the labory culture of snail intermediate hosts of Shistosoma haematobium. Ann. Trop. med. Parasit., 1960, 54, 3, 333-37.

4. DAYNES (P.). - La Distomatose à Madagascar - Cycle de Fasciola gigantica. Rev. Elev. Méd. Vét. Pays trop., 1967, 20, 4, 557-62.

5. DINNIK (J. A.) (a). - The snail hosts of certain Paramphistomidae and Gastrothylacidae (Trematoda) discovered by the late Dr P. L. Le Roux in africa. J. Helm. 1965. 39, 2/3, 141-150.

6. DINNIK (J. A.) and DINNIK (N. N.) (b). The Schistosomes of domestic ruminants in Eastern africa. Bull. epizoot. Dis. Afr., 1965. $13,4,341-59$.

7. GAUD (J.). ARFAA (F.) et ZEINI (A.). Observations sur la biologie de Bulinus truncatus au Khouzistan (Iran). Annis Parasit. Hum. comp., 1962, 37, 3, 232-75.

8. GERMAIN (L.). - La faune malacologique du lac Tchad et des pays bas du Tchad. Archs Mus. natr. Hist. nat. Poris, 1935, 12, Ser. G. 389-99.

9. GHANI (A.F. A.). - Biological observations on Lymnaea cailliaudi snail and its breeding in the laboratory. Agr. Res. Rev. Cairo, 1960, 38, 188.

10. GRABER (M.). - Helminthes et Helminthiases - Bilan d'activité. Rop. Ann. Laboratoire de Forcha - Fort-Lamy, 1967, t. III, 192 p.

11. GRETILLAT (S.). - Nature ef particularités biologiques du Schistosome agent causal de la bilharziose génito-urinaire et de la Bilharziose des ruminants domestiques de l'Afrique de l'Ouest. 7e Cong. Int. Med. trop., Rio de Janeiro, in Revue Elev. Méd. Vét. Pays trop., $1963,16,4,554-6$.

12. KENDALL (S. B.) and PARFIT (J. W.), - The life - history of some vectors of Fasciola gigantica under laboratory conditions. Ann. trop. med. Parosit., 1965, 59, 1, 10-16.

13. LE ROUX (P. L.). - Life - cycle of Gas- 
trodiscus aegyptiacus (Cobbold, 1876). Trans. R. Soc. Trop. Med. Hyg., 1958, 52, 1, 14-5.

14. LEVEQUE (C.). - Mollusques aquatiques de la zone est du Lac Tchad. (a). Bull. Inst. fr. Afr. noire, 1967, 29, ser. A, 4, 1494-1532.

15. LEVEQUE (C.). - Biologie de Bulinus forskali dans les mares temporaires de la région de Fort-Lamy (b). ORSTOM - Fort-Lamy, 1967, ronéo, $8 \mathrm{p}$.

16. MANDAHL-BARTH (G.). - Les hôtes interm médiaires de Schistosomes. OMS, ser. monogr., 1959, no 37, 95 p., 25 fig., 60 pl.

17. MANDHAL-BARTH (G.). - Key to identification of East and central african freshwater snails of medical and Veterinary importance. Bull. Wid. Heth. Org., 1962, 27, 1, $135-50$.

18. MANDAHL-BARTH (G.). - The species of the genus Bulinus intermediate host of Schistosoma. Bull. WId. Heth. Org., 1965, 33, 1, 33-44.
19. PORTER (A.). - The life - history of the African sheep and cattle fluke, Fasciola gigantica. S. Afr. J. Sci., 1920-21, 17, 126-30.

20. STANDEN (O. D.). - Experimental Shistosomiasis I the culture of the snail vector Planorbis boissyi and Bulinus truncatus. Ann. trop. Med. Porosit., 1949, 43-13.

21. SWART (P. J.) and REINECKE (R. K.).Studies on Paramphistomiasis I. Propagation of Bulinus tropicus Krauss 1848. Onderstepoort J. Vet. Res., 1962, 29, 2, 183-187.

22. WARD (P. A.), TRAVIS (D.) and RUE (R. E.). - Methods of establishing and maintaining snails in the laboratory. Nat. Inst. Heth. Bull., 1947, 189, 70.

23. FRANC (A.). - Classe des Gasteropodes Sous-classe des pulmonés. In traité de Zoologie de Grasse 1968, t. $V$, fasc. 3, 325-60. Vigot frères, Paris.

24. GRETILLAT (S.). - Rapport, Dakar, I. E.M.V.T., 1964, 17 p. 